\title{
Modelo de gerenciamento de materiais na construção civil utilizando avaliação multicritério
}

\author{
Nadia Kelner Szajubok \\ Luciana Hazin Alencar \\ Adiel Teixeira de Almieida
}

UFPE

\begin{abstract}
Resumo
Este trabalho apresenta um modelo mais estruturado para o processo decisório no que diz respeito à gestão de materiais na construção civil. O processo de gestão de materiais tem assumido grande relevância, apontando seu caráter estratégico de potencial redutor de custos e propulsor da inovação tecnológica e organizacional das empresas de construção. A questão básica a ser tratada é a forma como pode ser realizada uma classificação dos materiais à luz de múltiplos critérios estabelecidos. Com o intuito de classificar os materiais atendendo aos critérios definidos pelo decisor, um modelo de gestão de materiais apoiado pelo uso do método multicritério ELECTRE TRI é apresentado e aplicado em uma edificação residencial vertical.
\end{abstract}

Palavras-chave

Construção civil, gestão de materiais, processo decisório, ELECTRE TRI.

\section{Materials management model in the civil construction based on multicriteria evaluation}

\begin{abstract}
This paper discusses a more structured model for the decision-making process with regard to management material process, through the application of the multicriteria decision aid method as a support in one model. The management material process has assumed great relevance due to its strategic role on reducing costs and pushing up the construction companies' technological and organizational innovation. The basic question to be considered is how the material classification can be done using multiple criteria. The model was applied in a building site, through the application of the ELECTRE TRI multicriteria decision aid method, with the aim of categorizing the material in three classes.
\end{abstract}

Key words

Civil construction, material management, decision process, ELECTRE TRI. 


\section{INTRODUĈ̣̃O}

A indústria da Construção Civil tem grande importância socioeconômica e estratégica para o desenvolvimento do País. Sendo um setor dos mais dinâmicos da economia brasileira, a cadeia produtiva da construção civil teve participação de 7,1 \% do Produto Interno Bruto (PIB) nacional em 2003, segundo o Instituto Brasileiro de Geografia e Estatística (IBGE).

Tendo seus lucros assegurados de forma permanente, os empresários não dispensaram o devido apreço ao gerenciamento da cadeia de suprimentos no ambiente produtivo. Porém, com o transcorrer do século XX, a situação econômica brasileira foi sendo, aos poucos, levada a uma integração universal, decorrente da globalização. técnicas ABC. Recentemente, os sistemas de administração MRP (Manufacturing Resource Planning) e ERP (Enterprise Resource Planning) foram adicionados.

Essas abordagens para o controle de inventário têm provocado um grande valor na determinação de parâmetros de estoque e planejamento de recursos, tendo sido seus valores questionados com respeito a problemas práticos de controle de inventário. Adicionalmente, vários autores afirmam que muitos métodos quantitativos existentes na administração de produção e pesquisa operacional são insuficientes para enfrentar a complexidade das organizações (HAYES, 1998; LOVEJOY, 1998; MACHUCA, 1998). Esses autores oferecem um amplo escopo na administração de produções, incluindo aspectos qualitativos e link com outras áreas da organização.

Assim, é importante analisar o problema de estoques em construção civil, considerando-se os critérios qualitativos e quantitativos para a confecção de um modelo de curva $\mathrm{ABC}$, utilizando métodos multicritério de apoio à decisão. A questão básica a ser tratada é a forma como pode ser realizada uma classificação dos materiais à luz de múltiplos critérios.

Deste modo, o objetivo deste

Ao longo dos anos, a área técnico-estrutural foi colocada em primeiro plano na construção civil pela sua real importância, e à área de suprimentos não foi dada a devida importância, apesar de os materiais representarem um elevado percentual nos custos de construção. Eis que o suprimento é a fase da aquisição, fabricação, transporte e entrega, na obra, de todos os equipamentos, estruturas e materiais em geral necessários à construção e indispensáveis à execução do empreendimento.

Um sistema de gerência dos materiais inclui as funções principais de identificar, adquirir, distribuir e dispor dos materiais necessitados em um projeto de construção. Seus objetivos mais importantes são assegurar que materiais de qualidade estarão disponíveis onde foram solicitados; obter o melhor valor para compras de materiais; fornecer eficientemente baixo custo de transporte, segurança e armazenamento dos materiais dentro dos locais de construção; além de reduzir o excesso de materiais ao nível mais baixo possível. Nesse sentido, uma boa gestão de estoques garantirá uma série de benefícios, tais como diminuição da hora improdutiva, redução do desperdício, produção constante e, conseqüentemente, aumento do lucro.

$\mathrm{O}$ controle de estoques tem sido alvo de muitos estudos reportados na literatura. Como resultado, uma larga coleção de conceitos e técnicas estão disponíveis para o controle de estoques, como os modelos estocásticos para a determinação da quantidade de pedido, técnicas de projeção de demanda e trabalho é apresentar um modelo mais estruturado para o processo decisório, no que diz respeito à gestão de materiais na construção civil, através da aplicação do método de apoio a decisão ELECTRE TRI, visando classificar os materiais de uma obra em três categorias, para cada uma das quais devem ser adotados procedimentos de políticas de estoques, assim como auxiliar na análise e estruturação da complexidade do problema de gestão de materiais.

\section{GERENCIAMENTO DA CADEIA DE SUPRIMENTOS NA CONSTRUC̣ÃO CIVIL}

Walsh et al. (2004) definem gerenciamento da cadeia de suprimentos como a prática de um grupo de empresas e de indivíduos trabalhando de forma colaborativa em uma rede de processos interrelacionados, estruturados para melhor satisfazer as necessidades do usuário final.

Em termos de estrutura e função, Vrijhoef e Koskela (2000) caracterizam a cadeia de suprimentos na construção civil da seguinte forma:

- É uma cadeia de suprimentos convergente, direcionando todos os materiais para o canteiro de obras, onde o produto final é produzido.

- É, excluindo raras exceções, uma cadeia de suprimentos temporária, produzindo projetos de construção únicos.

- É uma típica cadeia de suprimentos make-to-order. Há pouca repetição, com raras exceções. 
Tais características exercem um impacto no gerenciamento da cadeia de suprimentos. Diante disso, Vrijhoef \& Koskela (2000) estabeleceram quatro regras que podem ser reconhecidas a depender do foco do gerenciamento: na cadeia de suprimentos, no canteiro de obras ou em ambos, conforme apresentado na figura abaixo.

Tommelein (1998), por sua vez, atribui a dificuldade de aplicar os conceitos da cadeia de suprimentos ao problema de "matching" encontrado na construção: uma significante parte dos materiais entregues no canteiro de obras deve ser precisamente combinada ao seu local de instalação. No entanto, construtores procuram ter os materiais no seu canteiro mais cedo do que possível, não fornecendo a devida instalação planejada para tais materiais.

Davis et al. (2001) definem os estoques como a quantificação de qualquer item ou recurso usado em uma organização. No escopo completo, o estoque pode incluir entradas como itens humanos, financeiros, energia e equipamentos; físicos, tais como matéria-prima; saídas, como peças, componentes e produtos prontos; e estágios intermediários do processo, tais como produtos parcialmente acabados ou estoque em processo (ou estoque intermediário). A escolha de quais itens incluir no estoque depende da organização.
Na construção civil, são feitos estoques de materiais para a construção; logo, estoque de entrada.

Os principais objetivos operacionais dos estoques são cobrir mudanças previstas no suprimento e na demanda, proteger a produção contra incertezas e permitir produção ou compras econômicas.

Na construção civil, pode-se verificar o estoque de proteção como o de maior importância, cujo objetivo é compensar as incertezas entre fornecimento e demanda, pois existe um tempo entre o pedido ser feito e o produto chegar à obra, e nesse intervalo é necessário ter a matéria-prima para que não haja uma interrupção no processo construtivo. Portanto, esse tipo de estoque é o principal objetivo deste estudo.

A construção civil não se caracteriza por manter estoques a longo prazo, uma vez que se apóia na produção "puxada", em que os insumos para a execução das tarefas chegam à obra de acordo com a atividade a ser realizada. Entretanto, existe limitação quanto ao volume de material a ser entregue no canteiro de obras, devido à capacidade do veículo que realiza o transporte e às flutuações nos preços dos materiais. Portanto, ocorre a aquisição de insumos em quantidade maior do que seria utilizada em determinada atividade.

$\mathrm{O}$ gerenciamento do estoque na construção requer senso

Figura 1: As quatro regras do gerenciamento da cadeia de suprimentos na construção (adaptado de VRIJHOEF e KOSKELA, 2000).

Regra 1: foco na interface entre a cadeia de suprimentos e o canteiro de obras
Regra 2: foco na cadeia de suprimentos

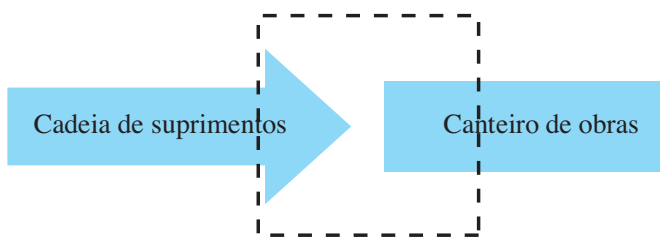

Regra 3: foco na transferência das atividades do canteiro de obras para a cadeia de suprimentos

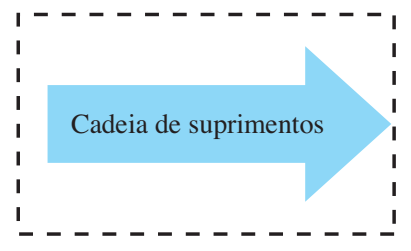

\section{Canteiro de obras}

Canteiro de obras

Regra 4: foco no gerenciamento integrado da cadeia de suprimentos e do canteiro de obras

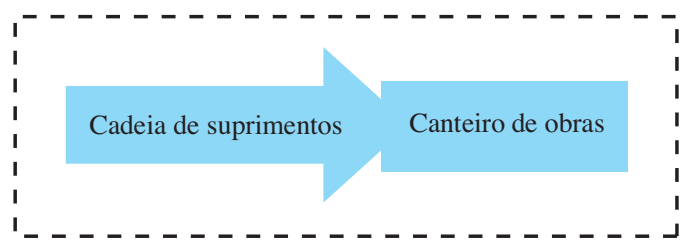


no balanço entre o desejo do construtor ter os materiais cedo no canteiro e os benefícios decorrentes da redução do estoque. Em um extremo, $100 \%$ de estoque antes de se iniciar o projeto maximiza a flexibilidade do trabalho da equipe e elimina a possibilidade de atraso na construção devido a atrasos de entrega. No entanto, tem-se o custo de manter o estoque, o risco de danificação ou perda dos materiais e a inflexibilidade em resposta a mudanças no projeto (WALSH et al. , 2004).

A escolha de um método para o controle de estoques é determinada em função de dois padrões básicos de consumo de um item ao longo do tempo, denominados de demanda dependente e independente. A demanda de um item é independente se depende das condições de mercado, fora do controle imediato da empresa; é dependente quando seu consumo puder ser programado internamente.

Os insumos na construção civil possuem uma demanda dependente do planejamento realizado previamente. Segundo Leite e Possamai (2001), o planejamento da programação de obras em engenharia implica, inicialmente, uma previsão das atividades a serem realizadas, dos recursos necessários, dos custos estimados, dos prazos e de tantos outros elementos importantes para a execução e para o acompanhamento da obra.

Verifica-se empiricamente que uma pequena parte de insumos é responsável pela maior parte dos investimentos. Uma análise $\mathrm{ABC}$ em estoques permite agrupar os materiais em três classes, de acordo com a ordem de prioridade:

- Classe A: grupo de materiais mais importantes, que deve ser controlado pela administração. Embora esses itens constituam apenas $20 \%$, ou menos, da quantidade dos itens em estoque, representam $70 \%$ a $80 \%$ do investimento.

- Classe B: grupo de itens em situação intermediária entre as classes A e C. Geralmente constitui $20 \%$ dos itens, correspondendo a $20 \%$ do valor total em estoque.

- Classe C: grupo de itens menos importantes, que merecem pouco controle por parte da administração de materiais, e representa em média $60 \%$ em quantidade e apenas $10 \%$ do investimento total.

Uma análise exclusiva $\mathrm{ABC}$ pode levar a distorções perigosas para a empresa, pois ela não considera a importância do item em relação ao sistema como um todo. Para resolver essa deficiência da análise por investimento, muitas empresas utilizam um conceito chamado criticidade dos itens de estoque. Criticidade é a avaliação dos itens quanto ao impacto que sua falta causará na operação da empresa, na imagem da empresa perante os clientes, na facilidade de substituição de um item por um outro e na velocidade de obsolescência

(MARTINS; ALT, 2000).

Alguns estudos mostram que, na prática, a tradicional análise $\mathrm{ABC}$ pode não fornecer uma boa classificação dos itens do estoque. Há muitos casos em que outros critérios, que não o custo, tornam-se relevantes na decisão da importância de um item em estoque. Tal problema multicritério de classificação de estoque tem

Analisar em profundidade milhares de itens num estoque é uma tarefa extremamente difícil e, na grande maioria das vezes, desnecessária. É conveniente que os itens mais importantes, segundo algum critério ou critérios, tenham prioridade sobre os menos importantes. Assim, economizam-se tempo e recursos (FRANCISCHINI; GURGEL, 2002).

$\mathrm{Na}$ construção civil, existe uma variedade muito grande de itens. Gerenciar os estoques de todos eles com a mesma atenção pode ser dispendioso, fazendo com que se encontre uma forma de dar atenção diferenciada a certos itens, adotando critérios que permitam distinguir claramente a importância do insumo segundo esses critérios.

Um dos critérios mais utilizados e importantes é o que considera o investimento aplicado a cada item, visto que qualquer economia feita nesses estoques significa disponibilidade de recursos para investimentos em outras necessidades da empresa. sido referenciado por alguns estudos na literatura (HAUTANIEMI; PIRTTILÄ, 1999; GUVENIR; EREL, 1998; PARTOVI; ANANDARAJAN, 2002; DUCHESSI et al., 1988; FLORES; WHYBARK, 1986).

Flores \& Whybark (1986) recomendaram considerar um ou mais critérios, como certeza de suprimento, taxa de obsolescência, avaliabilidade, substitutibilidade, lead time (tempo de reabastecimento) e impacto da falta do item. O número de categorias sob qualquer sistema de classificação não precisa ser limitado a três ( $\mathrm{A}, \mathrm{B}$ ou $\mathrm{C})$. Flores e Whybark (1987) notaram que categorias adicionais podem ajudar a analisar a política de estoque. As empresas podem criar supercategorias, subcategorias ou categorias baixas, sob as quais estabelecerá políticas de administração de estoque, sistema e métodos de controle.

Dependendo do tipo de indústria e da parte da organização que é considerada, os números de critérios para a 
classificação $\mathrm{ABC}$ podem ser vários.

MÉTODOS MULTICRITÉRIO DE APOIO À DECISÃO

O escopo deste trabalho é o desenvolvimento de um modelo de controle de estoques, sob a ótica do apoio multicritério à decisão. Essa área do conhecimento possui um amplo conjunto de ferramentas cujo objetivo é dar suporte a um indivíduo ou grupo de pessoas (decisor) no desenvolvimento de um processo de decisão.

Quando modelamos um problema de decisão multicritério, várias problemáticas podem ser consideradas. Segundo Roy (1996), as problemáticas podem ser classificadas como de escolha, classificação, ordenação e descrição.

A problemática de escolha (P. $\alpha$ ) ajuda a escolher um subconjunto A' de A, tão pequeno quanto possível, direcionando a investigação para encontrar a "melhor" ação ou desenvolver um processo de seleção. O resultado dessa problemática indica a decisão que poderia ser tomada ou propõe uma seleção baseada numa metodologia que pode ser usada repetidamente para identificar as melhores ações. A problemática de classificação (P. $\beta$ ) consiste em formular o problema de decisão de tal forma que distribua cada alternativa de A para uma categoria predefinida. A distribuição de uma alternativa $a$ para sua categoria apropriada é devida ao valor real intrínseco de $a$ (e não da comparação de $a$ com outras alternativas de A).

A problemática de ordenação (P. $\gamma$ ) ajuda a ordenar as ações em ordem decrescente de preferência, classificando as ações de A, determinando uma ordem definida dos subconjuntos das ações de A, que podem ser consideradas "suficientemente satisfatórias", tendo como base o modelo de preferências, sendo essa ordenação completa ou parcial.

A problemática de descrição (P. $\delta$ ) consiste em descrever as ações e as suas conseqüências em uma maneira formalizada e sistemática, ou desenvolver um procedimento cognitivo.

Vários métodos multicritério de apoio à decisão são encontrados na literatura (GOMES et al. , 2002; GOMES et al., 2004; ALMEIDA; COSTA, 2003). Para Vincke (1992), esse campo de estudo possui duas linhas principais: a Escola Francesa e a Escola Americana. A família dos métodos ELECTRE (Elimination and Choice Translating Algorithm) constitui-se em um das mais citadas da Escola Francesa. Neste trabalho será utilizado o método ELECTRE TRI.

A descrição do método ELECTRE TRI é apresentada no apêndice deste trabalho.

\section{DESCRIĈ̣̃O DO MODELO}

O modelo ora proposto recomenda que sejam classifica- dos os itens do estoque de tal forma que sejam estabelecidas classes de artigos. Tal procedimento vem a facilitar não só a tomada das decisões, a respeito da política de estoques, a qual deverá ser sempre a mesma para cada classe, como auxiliar na análise e estruturação da complexidade do problema de gestão de materiais.

Neste trabalho, o modelo é aplicado à indústria da construção civil. No entanto, pode ser utilizado em qualquer outra atividade econômica.

O modelo segue, portanto, as etapas ilustradas na Figura 2.

Em um mercado altamente competitivo, as construtoras (particularmente as empresas pequenas) podem encontrar dificuldades para realizar um moderno sistema de gerência dos materiais. Não obstante, os benefícios a longo prazo são significativos. Sistemas bons de administração de materiais são essenciais para aquelas companhias que querem perma- necer competitivas no futuro.

Alguns estudos no campo da administração de materiais concluíram que um efetivo sistema de administração de materiais irá resultar numa melhora de $6 \%$ a $8 \%$ na produtividade, aumento do fluxo de caixa, redução do volume de excesso de materiais, redução de recursos humanos para a administração de materiais, melhora na performance do fornecedor, redução física do armazém, na realização de compras com descontos, além da minimização do impacto de mudança de pedidos e diminuição dos projetos atrasados (Construction Industry Institute, 1988).

\section{EXEMPLO ILUSTRATIVO}

A aplicação do modelo de gerenciamento de materiais foi realizada numa empresa construtora e incorporadora certificada com ISO 9001/2000 (ABNT, 2000) com vinte anos de mercado. Encontra-se sediada no Recife e tem como área de atuação os mercados público e privado.

A empresa é classificada como de médio porte, segundo o Sebrae (2004). Uma característica importante da empresa é que, nos últimos dois anos, vem implantando um sistema de gestão da qualidade e vem encontrando dificuldades no processo de suprimentos, por não ter um sistema de informação interligado entre contas a pagar, orçamento e pedidos.

As principais funções relacionadas com o objeto de es- 
tudo são os departamentos de planejamento, orçamento e custos, obras e suprimentos. O departamento de orçamento e custos encarrega-se principalmente da elaboração de orçamentos para obras públicas (participação de licitações) e obras privadas. Mensalmente são avaliados os custos da obra e é feita uma comparação com o que foi orçado. Já o departamento de obras é encarregado da administração da obra. Cabe ao engenheiro da obra controlar a realização da obra segundo os projetos e com qualidade. Ele é o responsável por encaminhar os pedidos de materiais à central de suprimentos e está autorizado a efetuar compras no caso de existir alguma urgência na obra. Em relação ao departamento de suprimentos, é caracterizado por ser centralizado.

No caso da obra alvo deste estudo, ela é composta de seis blocos, tendo cada um quatro pavimentos assim distribuídos: um térreo e três pavimentos-tipo. Cada pavimento-tipo possui oito apartamentos, medindo cada um $34 \mathrm{~m}^{2}$.

\section{Estruturação do problema}

Para a realização da caracterização do problema, alguns dados deverão ser coletados da empresa para que, com essas informações, seja possível avaliar as alternativas à luz de cada um dos critérios estabelecidos.

Em construção civil, são utilizadas normalmente três práticas gerenciais relativas aos materiais:

- Para materiais mais simples do ponto de vista de tempo de entrega, custo e impacto de falta, adota-se a prática de comprá-los em grande quantidade e estocá-los na obra, justamente pela simplicidade que os materiais apresentam, não exigindo do engenheiro uma atenção constante.

- Há os materiais que são mais complicados, seja pelo alto valor, seja pelo longo tempo de entrega, e/ou dependência deles em várias atividades, de maneira que sua falta causa interrupção na obra, o que faz com que o engenheiro estude caso a caso o sistema de compra que será utilizado para cada material, de acordo com os empecilhos que esses materiais possuem, e daí demandam uma atenção constante do engenheiro.

- Existem, igualmente, os materiais que estão entre esses dois casos e que são pedidos de acordo com a fase da obra e a necessidade.

Pode-se verificar que, na prática, conta-se com três categorias para a classificação dos materiais, o que irá determinar a forma como o engenheiro definirá as políticas de compras,

Figura 2: Modelo de classificação de itens de estoque.

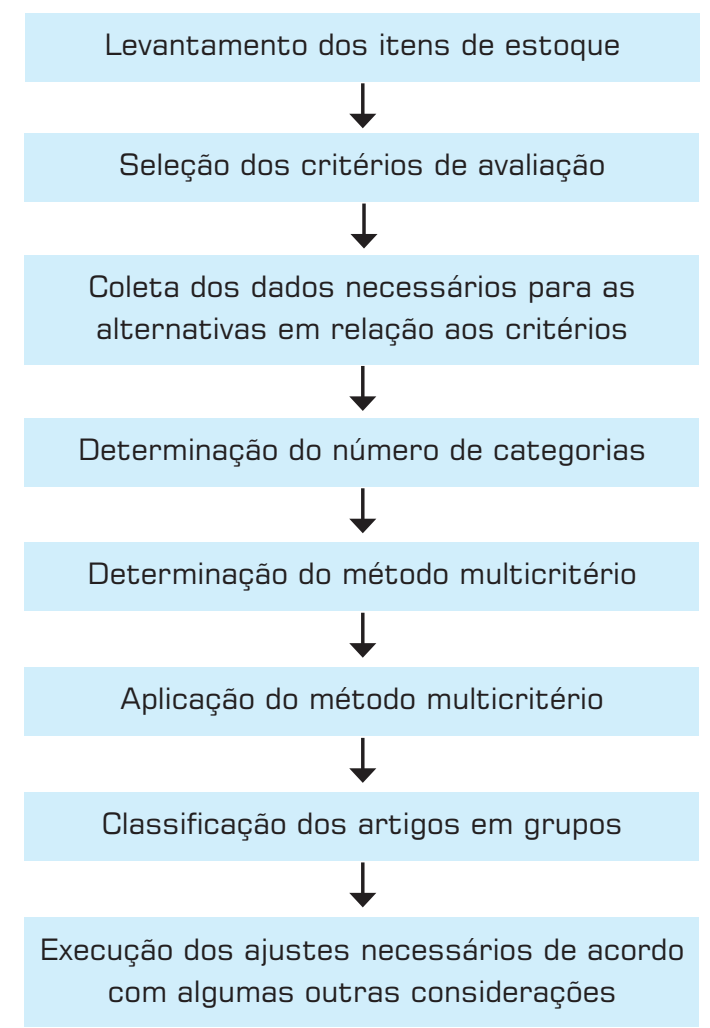


sendo uma categoria correspondente aos materiais "mais importantes" e que exigem atenção enorme do engenheiro, os materiais "menos importantes" e os que ficam no "nível intermediário".

Vale destacar que o escopo do trabalho prevê uma visão macro dos materiais, dividindo-os em três classes sob a ótica da visão multicritério. Posteriormente, há de se fazer um estudo micro de cada uma dessas classes para a análise das políticas de reposição que serão adotadas respondendo às perguntas do quando comprar e do quanto comprar.

O detalhamento desse trabalho está no nível tático, de extrema importância para o gerenciamento dos materiais da obra, pois é a partir dessa categorização que poderá ser analisada a política a ser adotada, a qual compreende um trabalho de nível operacional.

Então, de acordo com o que foi adotado na prática, os materiais serão qualificados pelo ELECTRE TRI em três grupos, mas nada impede que, num caso particular, mais classes sejam adicionadas para uma obra específica ou para um outro caso fora do contexto da construção civil.

\section{- Descrição do problema}

A responsabilidade pelo planejamento da produção e pela requisição dos materiais na obra é do engenheiro.

Normalmente, existem reuniões semanais com o diretor técnico e os engenheiros para revisar as programações das obras e avaliar o cronograma. Além disso, nas obras é verificado o avanço das atividades pelo engenheiro e analisado o estoque com o almoxarife para a programação de compras. O que ocorre é que, diariamente, são solicitados pedidos de compras de materiais. O problema desses pedidos diários é que, constantemente, os materiais requisitados são necessários na obra com urgência, o que ocasiona exceções no processo de compra que é realizado diretamente pela obra, e/ou atraso na entrega do material, atrasando assim o cronograma. Não existe, portanto, uma sistemática de controle de materiais.

Quanto ao sistema informatizado que dá suporte ao processo de suprimentos, embora as áreas de orçamento e controle de custos, suprimentos e planejamento de obra precisem de informações comuns, os sistemas não são interligados, dificultando a troca de informações.

\section{Aplicação do Modelo}

A seguir serão apresentadas as etapas da aplicação do modelo.

\section{- Levantamento dos materiais}

Para o levantamento dos materiais, foram avaliadas as atividades da obra com as respectivas composições de custos unitários, e daí foram retirados os insumos que serão classificados.

\section{- Avaliação das alternativas}

As avaliações das alternativas em relação aos critérios, tanto em valor absoluto como em importância relativa, merecem uma atenção especial, por se tratar do instrumento pelo qual são gerados os dados para o modelo e em que as alternativas manifestam suas características, representando os aspectos intrínsecos de cada sistema.

Os critérios considerados foram Custo do Investimento (CI), expresso em medidas monetárias; Lead Time (LT), considerado em dias; e Impacto de Falta (IF), avaliado pela quantidade de atividades a que o material está relacionado.

A partir dos itens avaliados de forma quantitativa, foi elaborada a Tabela 1, que se encontra anexa. Nela está a relação de materiais que serão utilizados para classificação, assim como a avaliação de cada uma das alternativas para cada critério considerado.

\section{- Aplicação do método ELECTRE TRI}

A construção do modelo para o ELECTRE TRI foi feita em cinco etapas:

1. Definição dos perfis $g_{j}\left(b_{h}\right)$ que representam os limites superiores das classes 1 a 3 . A escolha desses perfis foi feita pelo decisor, no caso o engenheiro da obra. A Tabela

Figura 4: Definição dos perfis do ELECTRE TRI.

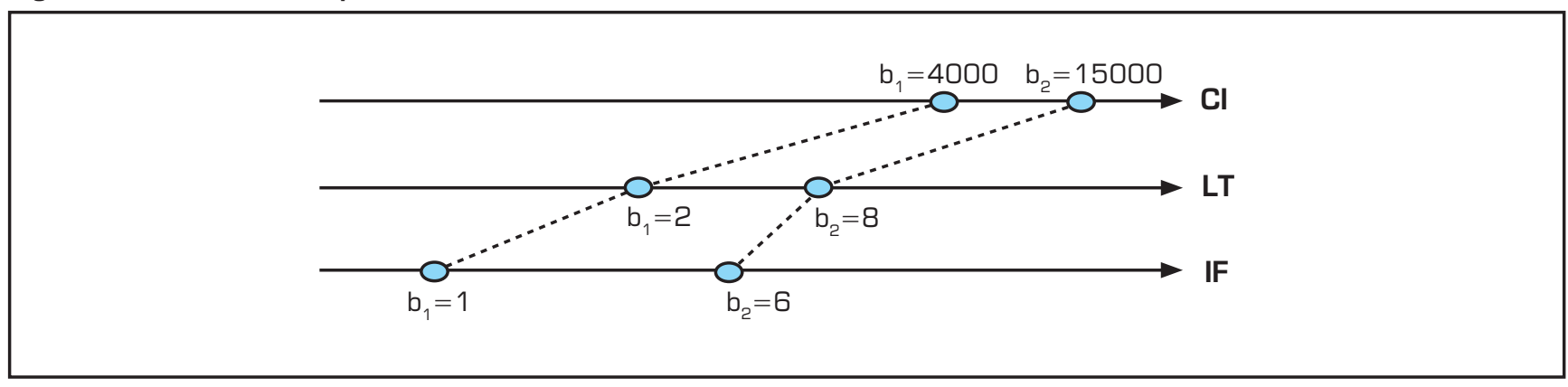


2 mostra os perfis e a Figura 4 representa essas classes.

2. Conjunto de coeficientes pesos $\left(\mathrm{j}_{1}, \mathrm{j}_{2}, \ldots \mathrm{j}_{\mathrm{m}}\right)$. É necessária a informação intercritério, correspondente à importância relativa entre os critérios, chamada de peso. A metodologia de atribuição de pesos empregada foi a de atribuição direta. Esses pesos podem ser vistos na Tabela 3.

3. Limites de indiferença $\left(\mathrm{q}_{\mathrm{j}}\left(\mathrm{b}_{\mathrm{h}}\right)\right)$ e preferência $\left(\mathrm{p}_{\mathrm{j}}\left(\mathrm{b}_{\mathrm{h}}\right)\right)$ : os valores dos limiares de preferência e indiferença para os critérios Lead Time e Impacto de falta foram considerados iguais a zero, pois não são aplicáveis ao tipo de escala utilizada. Isso equivale a usar critérios verdade ao invés de pseudocritérios para tais critérios. Para o critério Custo de Investimento aplica-se um percentual de $20 \%$ sobre o valor de $b_{h}$ para a definição do limiar de preferência, e um percentual de $10 \%$ para a definição do limiar de indiferença.

4. Limiares de veto $\mathrm{v}_{\mathrm{j}}\left(\mathrm{b}_{\mathrm{h}}\right)$ : o limiar de veto para todos os critérios foi definido como $30 \%$ do valor de $\mathrm{g}(\mathrm{b})$.

$\mathrm{v}=0,3 *^{\mathrm{g}}(\mathrm{b})$ para $\mathrm{CI}, \mathrm{LT}, \mathrm{IF}$

O conjunto de limiares de veto estão definidos na Tabela 4 abaixo:

5. Nível de corte $\lambda=0,5$

A escolha desse valor foi feita com base na análise de sensibilidade, de modo que a classificação fosse a melhor possível.

Depois de definidos os parâmetros, passa-se para a etapa dos cálculos, para que o método possa estabelecer

Tabela 2: Matriz dos limites das classes gj(bh).

\begin{tabular}{|l|c|c|c|}
\hline & CI (R\$) & LT(DIAS) & IF \\
\hline b1 & 4.000 & 2 & 1 \\
\hline b2 & 15.000 & 8 & 6 \\
\hline
\end{tabular}

Tabela 3: Critérios de avaliação e seus respectivos pesos

\begin{tabular}{|l|c|}
\hline \multicolumn{1}{|c|}{ CRITÉRIO } & PESO \\
\hline Custo do Investimento (CI) & 0,25 \\
\hline Lead Time (LT) & 0,35 \\
\hline Impacto de Falta (IF) & 0,4 \\
\hline
\end{tabular}

Tabela 4 - Matriz dos limiares de veto j(bh)

\begin{tabular}{|l|c|c|c|}
\hline & GI & LT & IF \\
\hline$j(b 1)$ & 1.200 & 0,6 & 0,3 \\
\hline$j(b 2)$ & 4.500 & 2,4 & 1,8 \\
\hline
\end{tabular}

uma relação de sobreclassificação entre uma alternativa $a$ e uma alternativa de referência $b_{h}$. Para essa finalidade, foram calculados os índices de concordância por critério - $\mathrm{c}_{\mathrm{j}}\left(a, b_{h}\right)$ e $\mathrm{c}_{j}\left(b_{h}, a\right)$, de concordância global - C $\left(a, b_{h}\right)$ e $\mathrm{C}\left(b_{h}, a\right)$, de discordância por critério - $\mathrm{d}_{j}\left(a, b_{h}\right)$ e $\mathrm{d}_{j}\left(b_{h}, a\right)$, de credibilidade $\alpha\left(a, b_{h}\right)$. Com os valores de $\alpha\left(a, b_{h}\right), \alpha\left(b_{h}, a\right)$ e $\lambda$ determinam-se as situações de preferência entre $a$ e $b_{\mathrm{h}}$.

Dados esses parâmetros, os materiais são classificados em três classes mediante os procedimentos otimistas e pessimistas do ELECTRE TRI. A partir dessa classificação é que se torna possível analisar a prática gerencial a ser adotada para políticas de reposição de cada uma das classes de materiais. A Tabela 5 com o resultado da aplicação do ELECTRE TRI encontra-se anexa. Alguns estudos apresentam um maior detalhamento do método ELECTRE TRI (MIRANDA; ALMEIDA, 2003; LOURENÇO; COSTA, 2003).

\section{- Análise de sensibilidade}

Para analisar a influência do comportamento do modelo quanto às variações impostas, foi feita uma análise de sensibilidade dos pesos dos principais critérios do modelo e dos parâmetros envolvidos, tais como: os limites de preferência e indiferença, os limiares de veto, o nível de corte e os perfis que representam as classes.

Análise de sensibilidade 1: Variação no parâmetro $\lambda$ nível de corte de 0,5 para 0,6 .

Nessa primeira simulação, com $\lambda=0,6$ e considerando-se os perfis utilizados na primeira aplicação, obteve-se uma pequena variação do resultado. $\mathrm{O}$ material $\mathrm{A}_{61}$, que obteve conceito 3 e 2 nas avaliações otimistas e pessimistas, respectivamente ,na primeira aplicação, passou a ser incomparável. Isso se deve ao aumento da exigência para declarar a afirmação $a \mathrm{~S} b_{h}$, e o conseqüente aumento das relações de incomparabilidade.

Análise de sensibilidade 2: Variação nos pesos dos critérios.

Para se analisar a influência dos critérios na avaliação, foi feita uma análise de sensibilidade dos pesos dos principais critérios do modelo.

- Análise 1: diminuição de 20\% no critério impacto de falta:

\begin{tabular}{|c|c|c|c|}
\hline CRITÉBIO & GI & LT & IF \\
\hline Peso & 0,29 & 0,39 & 0,32 \\
\hline
\end{tabular}

- Análise 2: aumento de 20\% no critério impacto de falta:

\begin{tabular}{|c|c|c|c|}
\hline CRITÉERIO & CI & LT & IF \\
\hline Peso & 0,21 & 0,31 & 0,48 \\
\hline
\end{tabular}

O que pode ser observado é que, com o aumento de $20 \%$ 
desse critério, o material $\mathrm{A}_{61}$ torna-se incomparável, e $\mathrm{A}_{46}$, que antes havia recebido classificação 3 no procedimento pessimista, passou para 2.

Análise de sensibilidade 3: variação nos limites das classes em $10 \%$ para mais:

\begin{tabular}{|c|c|c|c|}
\hline & GI (R\$) & LT (DIAS) & IF \\
\hline$b_{1}$ & 4.400 & 2,2 & 1,1 \\
\hline$b_{2}$ & 16.500 & 8,8 & 6,6 \\
\hline
\end{tabular}

Houve uma grande variação dos resultados devido ao aumento da exigência para cada uma das classes. O material $\mathrm{A}_{61}$ torna-se incomparável com essa variação; o material $A_{1}$, que tinha recebido classificação 3 no procedimento otimista, passou para 2; os materiais $\mathrm{A}_{8}, \mathrm{~A}_{11}, \mathrm{~A}_{13}, \mathrm{~A}_{14}$, $\mathrm{A}_{21}, \mathrm{~A}_{31}, \mathrm{~A}_{33}, \mathrm{~A}_{37}, \mathrm{~A}_{39}, \mathrm{~A}_{48}, \mathrm{~A}_{49}, \mathrm{~A}_{65}, \mathrm{~A}_{73}$ e $\mathrm{A}_{79}$, que haviam recebido 2 no mesmo procedimento, passaram para 1; os materiais $\mathrm{A} 17$ e $\mathrm{A}_{77}$, que haviam recebido classificação 2 no procedimento pessimista, passaram para 1, e $A_{60}$ e $A_{46}$ de 3 passaram para 2 .

Análise de sensibilidade 4: diminuição e aumento em 10\% do valor do veto.

Não houve alteração dos resultados.

Análise de sensibilidade 5: aumento e diminuição em 10\% nos limiares p e q.

Não houve alteração dos resultados.

\section{- Aplicação da Curva ABC}

Para a maioria das obras, a curva ABC é o que é normalmente aplicado para o nível estratégico de administração de materiais.

A figura da curva $\mathrm{ABC}$ dos materiais, usando o critério de custo do investimento, que é o comumente utilizado pelas construtoras, é apresentada abaixo.

É interessante comparar a diferença entre a aplicação da curva $\mathrm{ABC}$ utilizando apenas um critério e o realizado neste trabalho, que faz uso dos critérios mais importantes desse setor, com o intuito de mostrar as distorções que o uso de apenas um critério pode causar no gerenciamento da obra, já que não considera a importância do item em relação ao sistema como um todo. Para essa comparação, foi utilizado o gráfico da Figura 6, em que podem ser vistas claramente as diferenças entre as classificações feitas pelos dois métodos.

Em média, 40\% dos materiais obtiveram uma classificação diferente.

Essa comparação foi feita avaliando-se o procedimento pessimista em relação aos parâmetros fornecidos pelo decisor.

Uma comparação entre o procedimento otimista e a curva $\mathrm{ABC}$ é feita na Figura 7. Quando comparados com essa avaliação, 73\% dos materiais são classificados de forma diferente.

A diferença em relação ao procedimento otimista é maior porque a maior parte da curva $\mathrm{ABC}$ consiste nos materiais de classe 1 . No procedimento otimista, essa é a classe com menor número de materiais, justamente por esse procedimento julgar os materiais para a classe $\mathrm{C}_{\mathrm{h}}$ apenas quando $\mathrm{b}_{\mathrm{h}}>a\left(\mathrm{~b}_{\mathrm{h}}\right.$ preferível a $a$ ), o que significa considerar o "melhor dos casos", daí o nome "otimista". Isso torna o material "melhor avaliado", isto é, classificado para uma categoria superior,

Figura 5: Curva ABC.

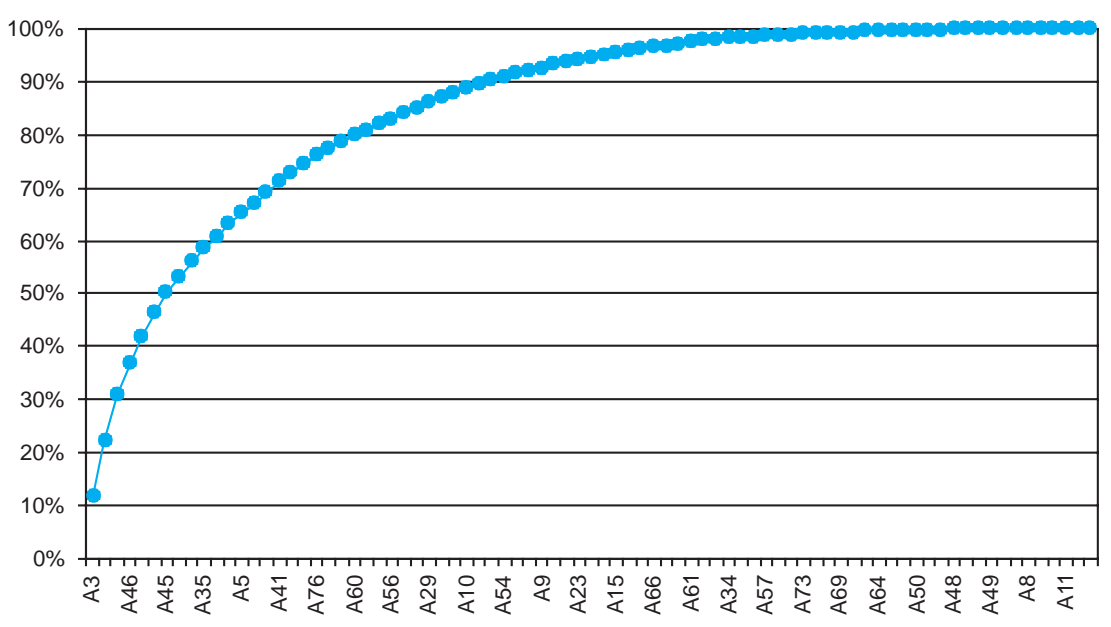


Figura 6: Comparativo entre a Classificação da Curva ABC e do Procedimento Pessimista realizado pelo ELECTRE TRI.

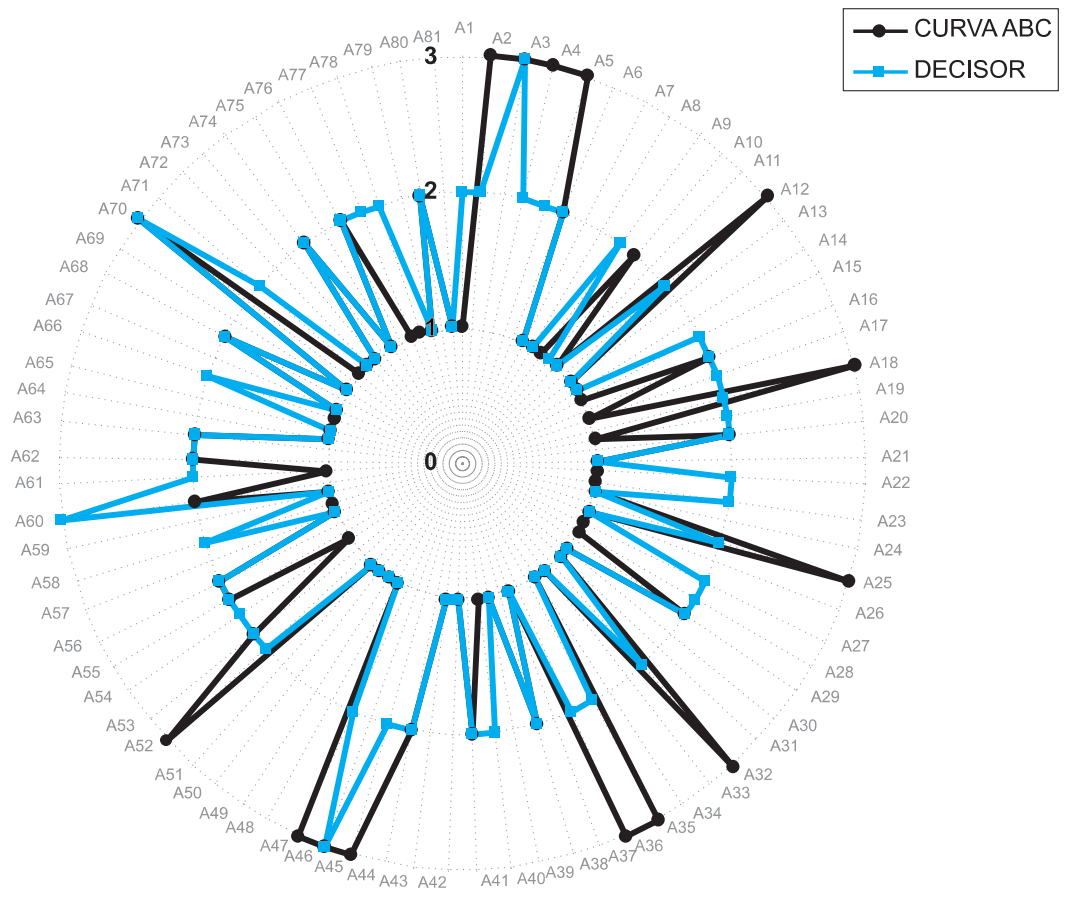

Figura 7: Comparativo entre a Classificação da Curva ABC e do Procedimento Otimista realizado pelo ELECTRE TRI.

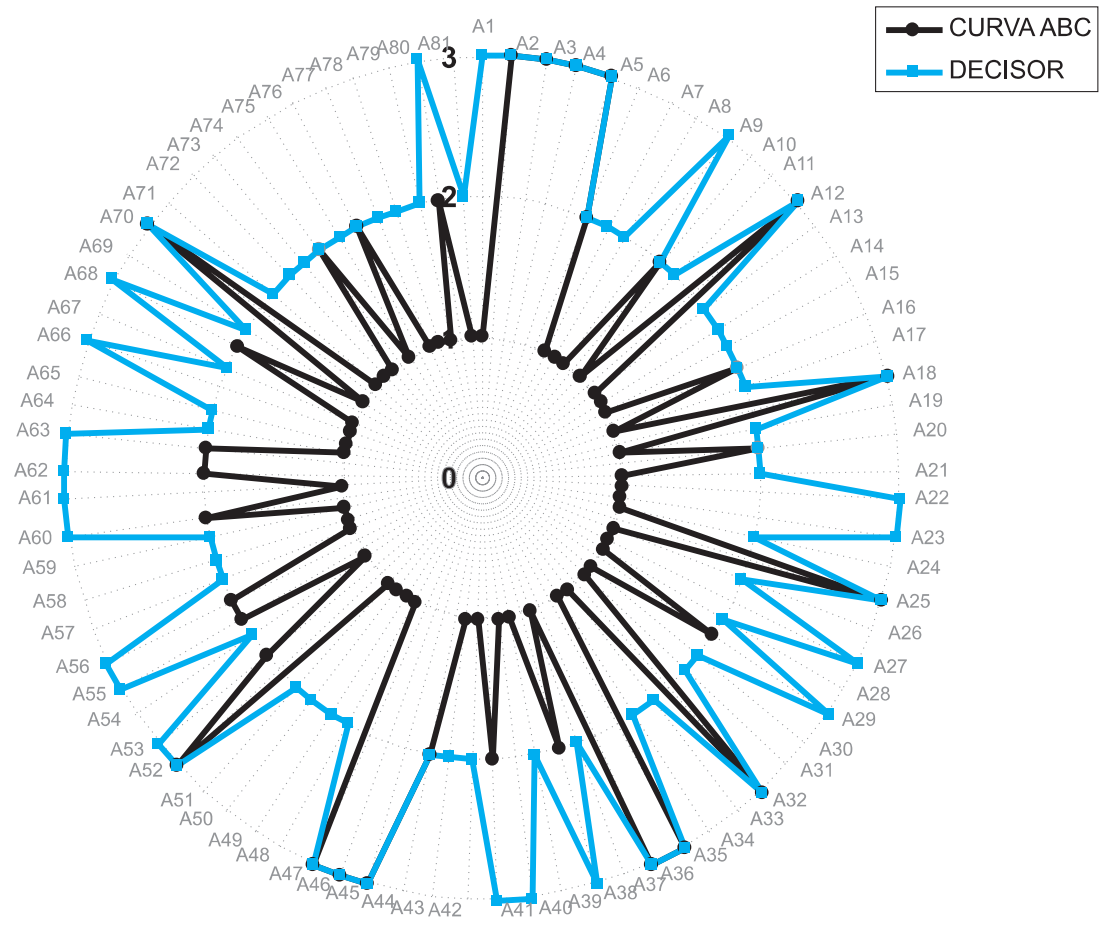


deixando a categoria 1 com poucos materiais. CONCLUSÕES

Este trabalho teve como objetivo apresentar um modelo mais estruturado para o gerenciamento de materiais, no intuito de ajudar na análise e estruturação da complexidade do problema em questão e de facilitar a tomada das decisões a respeito da política de estoques.

O problema de gerenciamento de estoques em construção civil foi discutido, apresentando as principais características do setor. Os principais modelos utilizados na literatura para esse tipo de problema foram pesquisados e adaptados para o problema em questão.

A abordagem outranking (sobreclassificação) foi a adotada, em especial o método ELECTRE TRI, que revelou um grande potencial de aplicação em problemas desse contexto, pois trata do tipo de problemática $\mathrm{P} \beta$ e apresenta resultados satisfatórios.

O modelo proposto apresentou uma visão geral do problema e foi aplicado em uma obra na Região Metropolitana do Recife, cuja construtora apresenta problemas para gerenciamento de estoque.

Um aspecto importante do estudo foi a construção da matriz de classificação, em que materiais (ações) utilizados foram obtidos da composição do custo unitário da obra e dos critérios de avaliação que, coerentes com o objetivo do problema, foram estabelecidos pelo engenheiro da obra (tomador de decisão). Assim, os fatores considerados na construção de cada critério de avaliação, bem como seus respectivos pesos, limiares de preferência, indiferença, veto e nível de corte, refletiram o conhecimento e a experiência do decisor na área.

Ressalte-se que não é tarefa fácil reunir uma multiplicidade de variáveis num único modelo de decisão. A prova disso é que os sistemas de Gestão de Estoques se baseiam exclusivamente em aspectos econômico-financeiros, mais precisamente na Curva $\mathrm{ABC}$.

Incorporar os múltiplos critérios no processo de Gestão de Estoques torna-se fundamental num momento em que as construtoras entram na concorrência global, com lucros cada vez menores, visto que os materiais representam $60 \%$ a $70 \%$ do valor global da obra. Posto isto, as políticas e objetivos estratégicos devem ser refletidos desde a definição de critérios de gerenciamento de materiais até as decisões de políticas de estoques.

Talvez a maior dificuldade na área de gerenciamento de estoques esteja em considerar aspectos que, a priori, não podem ser medidos monetariamente ou não sejam facilmente obtidos, mas que os decisores consideram implicitamente em suas tomadas de decisão de classificação de estoques para compras. E é justamente por negligenciar a relação entre "tomada de decisão" e "apoio à decisão" que aparece a principal dificuldade, qual seja, a de entender a decisão como um processo do qual fazem parte as características objetivas das "ações" e as perspectivas subjetivas dos "decisores", em que os modelos multicritérios num processo de construção, baseado na estruturação, apóiam a tomada de decisão.

Vale salientar que a aplicação realizada neste trabalho não deve nem pode ser generalizada. Cada aplicação apresentará um processo, uma avaliação, um resultado diferente, a qual poderá ser realizada em outras áreas que não a construção civil, e que poderá ser utilizada com o número de classes necessário para cada caso.

Dessa forma, o modelo desenvolvido classificou os materiais em três classes da obra, visando a um papel estratégico no setor de suprimentos em construção civil, o que constitui uma tendência nos dias atuais, mas que ainda não é uma

\section{Artigo recebido em 15/06/2005 Aprovado para publicação em 13/03/2006}

\section{- Referências Bibliográficas}

ALMEIDA, A. T.; COSTA, A. P. C. S. (Org.) Aplicações com métodos multicritério de apoio a decisão. Recife: Universitária, 2003.

ASSOCIAÇÃO BRASILEIRA DE NORMAS TÉCNICAS. NBR ISO 9001: Sistemas de Gestão da Qualidade - Requisitos. Rio de Janeiro, 2000.

CONSTRUCTION INDUSTRY INSTITUTE. Texas. Project Materials Management Primer. Bureau of Engineering Research Publication 7-2. University of Texas at Austin, nov. 1988.
DAVIS, M. M.; AQUILIANO, N. J.; CHASE, R. B. Fundamentos da Administração da Produção. 3. ed., Porto Alegre: Bookman, 2001. 598 p.

DUCHESSI, P.; TAYI, G. K.; LEVY, J. B. A conceptual approach for managing of spare parts. International Journal of Physical Distribution and Materials Management; $\mathrm{v}$. 18 , n. 5 , p. $8-15,1988$.

FLORES, B. E.; WHYBARK, D. C. Multiple criteria $\mathrm{ABC}$ analysis. International Journal of Operations and Production Management. v. 6, n. 3 , p. 38-46, 1986
FLORES, B. E.; WHYBARK, D. C. Implementing multiple criteria $A B C$ analysis. Journal of Operations Management, v. 7, n. l, p. 79-86, 1987

FRANCISCHINI, P. G.; GURGEL, F. A. Administração de Materiais e do Patrimônio. São Paulo: Pioneira Thomson, 2002.

GOMES, L. F. A. M; GOMES, C. F. S.; ALMEIDA, A. T. Tomada de decisão gerencial: enfoque multicritério. São Paulo: Atlas, 2002. 264 p.
GOMES, L. F. A. M.; ARAYA, M. C. G CARIGNANO, C. Tomada de decisões em cenários complexos: introdução aos métodos discretos do apoio multicritério à decisão. São Paulo: Pioneira, 2004. 168 p.

GUVENIR HA, EREL E. Multicriteria inventory classification using a genetic algorithm. European Journal of Operational Research, v. 105, p. 29-37, 1998.

HAUTANIEMI P.; PIRTTILÄ T. The choice of replenishment policies in an MRP environment. International Journal of Production Economics, v. 59, p. 85-92, 1999. 
HAYES, R. H. Developing POM faculties for the $21^{\text {st }}$ century. Production and Operations Management, [s.l.], v. 7, n. 2, p. $94-98,1998$.

INSTITUTO BRASILEIRO DE GEOGRAFIA E ESTATÍSTICA. Produto interno bruto. Disponível em: <http://www.ibge.gov. br $>$. Acesso em: 20/6/2004.

LEITE, M. O.; POSSAMAI, O. A utilização das curvas de aprendizagem no planejamento da construção civil. In: ENCONTRO NACIONAL DE ENGENHARIA DE PRODUÇÃO, 21, 2001. Anais... Santa Catarina, 2001, CD ROM.

LOURENÇO, R. P.; COSTA, J. P. Using ELECTRE TRI outranking methos to sort MOMILP nondominated solutions. European Journal of Operational Research, v. 153, n. 2, p. 271-289, 2004.

LOVEJOY, W. S. Integrated operations: a proposal for operations management teaching and research. Production and Operations Management. v. 7, n. 2, p. 106-124, 1998.

MACHUCA, J. A. D. Improving POM learning: systems thinking and transparent-box business simulators. Production and Operations Management, v. 7, n. 2, p. 210-227, 1998

MARTINS, P. G.; ALT, P. R. C. Administração de Materiais e Recursos Patrimoniais.2.ed. São Paulo: Saraiva, 2000.

MIRANDA, C. M. G.; ALMEIDA, A. T. Avaliação de pós-graduação com método ELECTRE TRI : o caso de Engenharias III da CAPES. Revista Produção, v. 13, n. 3, p. 101-112, 2003

MOUSSEAU, V.; SLOWINSKI, R.; ZIELNIEWICZ, P. A user-oriented implementation of the ELECTRE TRI method inte- grating preference elicitation support. Computers \& Operations Research, v. 27, $\mathrm{n}$. 7 , p. $757-777,2000$

MOUSSEAU, V.; DIAS, L. Valued outranking relations in ELECTRE providing manageable disaggregation procedures. European Journal of Operational Research, $\mathrm{v}$. 156, [s.n.], p. 467-482, 2004

PARTOVI, F. Y; ANANDARAJAN, M. Classifying inventory using an artificial neural network approach. Computers and Industrial Engineering, v. 41, p.389-404, 2002.

ROY, B. Multicriteria Methodology for Decision Aiding. Netherlands: Kluwer Academic Plublishers, 1996.

SERVICO BRASILEIRO DE APOIO ÀS MICRO E PEQUENAS EMPRESAS. Classificação do porte das empresas. Disponível em: <http://www.sebraesp.com.br/novo/ pesquisa/download/informacoes_basicas. doc > . Acesso em: 25/3/2004

TOMMELEIN, I. D. Pull driven scheduling for pipe-spool installation: simulation of a lean construction technique. Journal of construction engineering management, $\mathrm{v}$. 124, n. 4, p. 279-288, 1998.

VINCKE, P. Multicriteria decision-aid. Bruxelles: John Wiley \& Sons, 1992

VRIJHOEF, R.; KOSKELA, L. The four roles of supply chain management in construction. European Journal of Purchasing \& Supply Management, v. 6, n. 3-4, p.169178,2000

WALSH, K. D., J. C. HERSHAUER, et al. Strategic positioning of inventory to match demand in a capital projects supply chain. Journal of Construction Engineering and Management-Asce, v.130, n. 6, p. 818826,2004

\section{- Sobre os autores}

\section{Nadia Kelner Szajubok}

Universidade Federal de Pernambuco/UFPE

Consultora independente

Aluna Egressa do Mestrado de Engenharia de Produção - UFPE

E-mail: nkelner@iteci.com.br

\section{Luciana Hazin Alencar}

Universidade Federal de Pernambuco/UFPE

Grupo de Pesquisa em Sistema de Informação e Decisão - GPSID

Professora do Departamento de Engenharia de Produção

End.: Cx. Postal 7462 - CEP 50.630-970, Recife - PE

Tel./Fax: (81) 2126-8728 ramal 29

E-mail: lhazin@ufpe.br

\section{Adiel Teixeira de Almeida}

Universidade Federal de Pernambuco/UFPE

Grupo de Pesquisa em Sistema de Informação e Decisão - GPSID

Professor do Departamento de Engenharia de Produção e Coordenador do GPSID

End.: Cx. Postal 7462 - CEP 50.630-970, Recife - PE

Tel./Fax: (81) 2126-8728 ramal 29

E-mail: aalmeida@ufpe.br 
realidade completamente reconhecida no setor. 


\section{ANEXO}

Tabela 1: Matriz de avaliação das alternativas

\begin{tabular}{|c|c|c|c|c|}
\hline ALTERNATIVAS & CÓD. & CI (R\$) & LT (DIAS) & IF \\
\hline Aço CA-50 & $A_{1}$ & 4201,14 & 8 & 6 \\
\hline Aço CA-50 Armafer & $A_{2}$ & 22503,60 & 8 & 1 \\
\hline Aço CA-60 Armafer] & $A_{3}$ & 132723,13 & 8 & 13 \\
\hline Aço CA-60 Tela & $\mathrm{A}_{4}$ & 57128,94 & 8 & 1 \\
\hline Aço CA-60 Tela da Laje & $A_{5}$ & 23315,10 & 8 & 4 \\
\hline AGUA FRIA & $A_{6}$ & 11765,88 & 3 & 1 \\
\hline Aguarras mineral & $A_{7}$ & 660,35 & 2 & 4 \\
\hline $\begin{array}{l}\text { Arame Galvanizado } \\
\text { n } 12\end{array}$ & $A_{8}$ & 223,24 & 2 & 1 \\
\hline Arame recozido $\mathrm{n}^{0} 18$ & $A_{9}$ & 6117,65 & 2 & 25 \\
\hline Areia Média & $A_{10}$ & 8547,78 & 1 & 40 \\
\hline Argamassa Colante & $A_{11}$ & 12,45 & 2 & 1 \\
\hline Argamassa multiuso & $A_{12}$ & 21830,37 & 3 & 4 \\
\hline $\begin{array}{l}\text { Azulejo Branco } \\
15 \times 15 \mathrm{~cm}\end{array}$ & $A_{13}$ & 187,39 & 1 & 1 \\
\hline Barro de Aterro & $A_{14}$ & 10,56 & 2 & 1 \\
\hline Bloco “J" & $A_{15}$ & 4425,73 & 5 & 1 \\
\hline Bloco “U” 19cm & $A_{16}$ & 11503,17 & 5 & 1 \\
\hline Bloco "U" 7cm & $A_{17}$ & 3783,55 & 5 & 1 \\
\hline $\begin{array}{l}\text { Bloco Cerâmico } \\
\text { Estrutural }\end{array}$ & $A_{18}$ & 95872,04 & 5 & 1 \\
\hline $\begin{array}{l}\text { Bloco Cerâmico } \\
\text { Estrutural e=11cm }\end{array}$ & $A_{19}$ & 4964,54 & 5 & 1 \\
\hline $\begin{array}{l}\text { Bloco Cerâmico } \\
\text { Estrutural } e=14 \mathrm{~cm}\end{array}$ & $A_{20}$ & 9735,21 & 5 & 1 \\
\hline Bomba centrífuga & $A_{21}$ & 2010,00 & 2 & 1 \\
\hline Brita 19 & $A_{22}$ & 4100,00 & 2 & 16 \\
\hline Brita 25 & $A_{23}$ & 5136,89 & 2 & 17 \\
\hline Brita 38 & $A_{24}$ & 283,73 & 2 & 2 \\
\hline CABOS & $A_{25}$ & 25868,40 & 30 & 1 \\
\hline Cal Hidratada & $A_{26}$ & 1167,75 & 3 & 5 \\
\hline Centro MM & $A_{27}$ & 7200,00 & 30 & 3 \\
\hline $\begin{array}{l}\text { Chapa compensada de } \\
10 \mathrm{~mm}\end{array}$ & $A_{28}$ & 5775,00 & 3 & 1 \\
\hline $\begin{array}{l}\text { Chapa compensada de } \\
12 \mathrm{~mm}\end{array}$ & $A_{29}$ & 11018,01 & 3 & 14 \\
\hline $\begin{array}{l}\text { Chuveiro com haste } \\
\text { de plástico de } 3 / 4 " \text {, } \\
\text { Fortilit ou similar. }\end{array}$ & $A_{30}$ & 370,56 & 3 & 1 \\
\hline Cimento Branco & $A_{31}$ & 6,01 & 2 & 1 \\
\hline
\end{tabular}

\begin{tabular}{|c|c|c|c|c|}
\hline ALTERNATIVAS & CÓD. & 이 (R\$) & LT (DIAS) & IF \\
\hline Hidrômetro geral & $A_{43}$ & 480,00 & 3 & 1 \\
\hline Hidrômetro individ. & $A_{44}$ & 12480,00 & 3 & 1 \\
\hline Isopor & $A_{45}$ & 40564,80 & 3 & 1 \\
\hline Janela de Madeira & $A_{46}$ & 65212,13 & 40 & 5 \\
\hline Laje treliçada & $A_{47}$ & 114379,09 & 15 & 3 \\
\hline Liquido preparador & $A_{48}$ & 392,83 & 2 & 1 \\
\hline Liquido selador PVA & $A_{49}$ & 362,19 & 2 & 1 \\
\hline Ferragens para Tapume & $A_{39}$ & 1155,00 & 2 & 1 \\
\hline Fundo branco fosco & $A_{40}$ & 3395,93 & 15 & 3 \\
\hline $\begin{array}{l}\text { Grade de Madeira de } \\
\text { Lei }\end{array}$ & $A_{41}$ & 21672,00 & 8 & 4 \\
\hline $\begin{array}{l}\text { Grade de Proteção de } \\
\text { Ferro }\end{array}$ & $A_{42}$ & 270,00 & 8 & 1 \\
\hline Hidrômetro geral & $A_{43}$ & 480,00 & 3 & 1 \\
\hline Hidrômetro individual & $A_{44}$ & 12480,00 & 3 & 1 \\
\hline Isopor & $A_{45}$ & 40564,80 & 3 & 1 \\
\hline Janela de Madeira & $A_{46}$ & 65212,13 & 40 & 5 \\
\hline Laje treliçada & $A_{47}$ & 114379,09 & 15 & 3 \\
\hline Liquido preparador & $A_{48}$ & 392,83 & 2 & 1 \\
\hline Liquido selador PVA & $A_{49}$ & 362,19 & 2 & 1 \\
\hline Lixa de madeira $n^{\circ} 100$ & $A_{50}$ & 592,66 & 2 & 5 \\
\hline LOUÇA 1 & $A_{51}$ & 1196,16 & 8 & 1 \\
\hline LOUÇA 2 & $A_{52}$ & 33690,24 & 8 & 1 \\
\hline Madeira de Lei & $A_{53}$ & 8525,52 & 15 & 1 \\
\hline MAT ELETRICO 1 & $A_{54}$ & 7407,36 & 3 & 1 \\
\hline MAT ELETRICO 2 & $A_{55}$ & 19185,39 & 15 & 1 \\
\hline MAT ELETRICO 3 & $A_{56}$ & 11891,40 & 30 & 1 \\
\hline MD & $A_{57}$ & 1620,00 & 8 & 2 \\
\hline Placa de Gesso & $A_{58}$ & 6775,95 & 3 & 1 \\
\hline Placa de Obra & $A_{59}$ & 1200,00 & 8 & 1 \\
\hline $\begin{array}{l}\text { Pontalete de Louro } \\
\text { Rosa de 3"x3" }\end{array}$ & $A_{60}$ & 13805,58 & 7 & 14 \\
\hline $\begin{array}{l}\text { Porta de Madeira } \\
\text { Compensada } \\
0,60 \times 2,10 m\end{array}$ & $A_{61}$ & 3456,00 & 15 & 1 \\
\hline $\begin{array}{l}\text { Porta de Madeira } \\
\text { compensada } \\
0,80 \times 2,10 m\end{array}$ & $A_{62}$ & 10560,00 & 15 & 1 \\
\hline
\end{tabular}




\begin{tabular}{|l|c|c|c|c|}
\hline Cimento Portland & $\mathrm{A}_{32}$ & 53160,46 & 2 & 39 \\
\hline $\begin{array}{l}\text { Cumeeira de } \\
\text { fibrocimento }\end{array}$ & $\mathrm{A}_{33}$ & 411,38 & 1 & 1 \\
\hline Dobradiça & $\mathrm{A}_{34}$ & 2252,34 & 8 & 3 \\
\hline ESGOTO & $\mathrm{A}_{35}$ & 26485,44 & 3 & 1 \\
\hline Estronca de Madeira & $\mathrm{A}_{36}$ & 23572,04 & 7 & 3 \\
\hline $\begin{array}{l}\text { Extintor Pó Químico } \\
\text { 4kg }\end{array}$ & $\mathrm{A}_{37}$ & 1680,00 & 1 & 1 \\
\hline Fechadura Completa & $\mathrm{A}_{38}$ & 20046,60 & 30 & 3 \\
\hline $\begin{array}{l}\text { Ferragens para } \\
\text { Tapume }\end{array}$ & $\mathrm{A}_{39}$ & 1155,00 & 2 & 1 \\
\hline Fundo branco fosco & $\mathrm{A}_{40}$ & 3395,93 & 15 & 3 \\
\hline $\begin{array}{l}\text { Grade de Madeira de } \\
\text { Lei }\end{array}$ & $\mathrm{A}_{41}$ & 21672,00 & 8 & 4 \\
\hline $\begin{array}{l}\text { Grade de Proteção de } \\
\text { Ferro }\end{array}$ & $\mathrm{A}_{42}$ & 270,00 & 8 & 1 \\
\hline Hidrômetro geral & $\mathrm{A}_{43}$ & 480,00 & 3 & 1 \\
\hline Hidrômetro individual & $\mathrm{A}_{44}$ & 12480,00 & 3 & 1 \\
\hline Isopor & $\mathrm{A}_{45}$ & 40564,80 & 3 & 1 \\
\hline Janela de Madeira & $\mathrm{A}_{46}$ & 65212,13 & 40 & 5 \\
\hline Laje treliçada & $\mathrm{A}_{47}$ & 114379,09 & 15 & 3 \\
\hline Liquido preparador & $\mathrm{A}_{48}$ & 392,83 & 2 & 1 \\
\hline Liquido selador PVA & $\mathrm{A}_{49}$ & 362,19 & 2 & 1 \\
\hline & & & & 1 \\
\hline
\end{tabular}

\begin{tabular}{|l|c|c|c|c|}
\hline $\begin{array}{l}\text { Porta de Madeira de } \\
\text { Lei 0,80x2,10m }\end{array}$ & $A_{63}$ & 7711,20 & 15 & 2 \\
\hline $\begin{array}{l}\text { Poste de Concreto } \\
\text { armado 100/8 }\end{array}$ & $A_{64}$ & 810,00 & 3 & 1 \\
\hline Prego 15x15 & $A_{65}$ & 87,00 & 2 & 1 \\
\hline Prego 2 1/2"x10 & $A_{66}$ & 3991,30 & 2 & 22 \\
\hline Quadro Bomba & $A_{67}$ & 390,00 & 3 & 1 \\
\hline $\begin{array}{l}\text { Sarrafo de Pinho de } \\
\text { 1"x4" }\end{array}$ & $A_{68}$ & 12026,74 & 7 & 16 \\
\hline Sika ou Vedacit & $A_{69}$ & 1093,00 & 2 & 3 \\
\hline $\begin{array}{l}\text { Tabua de Louro Rosa } \\
\text { de 1"x12" }\end{array}$ & $\mathrm{A}_{70}$ & 32530,06 & 7 & 18 \\
\hline $\begin{array}{l}\text { Tabua de Louro Rosa } \\
\text { de 1"x6" }\end{array}$ & $\mathrm{A}_{71}$ & 4640,58 & 7 & 1 \\
\hline $\begin{array}{l}\text { Tabua de Louro Rosa } \\
\text { de 1"x9" }\end{array}$ & $\mathrm{A}_{72}$ & 2633,04 & 7 & 1 \\
\hline $\begin{array}{l}\text { Tampa para } \\
\text { Reservatório }\end{array}$ & $\mathrm{A}_{73}$ & 1170,00 & 1 & 1 \\
\hline Telha canal & $\mathrm{A}_{74}$ & 14190,34 & 2 & 2 \\
\hline $\begin{array}{l}\text { Telha Ond. de Fibrocim. } \\
\text { de 4mm }\end{array}$ & $\mathrm{A}_{75}$ & 928,20 & 3 & 1 \\
\hline Tij. Cerâm. (8 furos] & $\mathrm{A}_{76}$ & 16378,43 & 3 & 2 \\
\hline $\begin{array}{l}\text { Tijolo Comum } \\
\text { 5x10x20cm }\end{array}$ & $\mathrm{A}_{77}$ & 3685,82 & 2 & 3 \\
\hline Tinta esmalte & $\mathrm{A}_{78}$ & 5163,36 & 2 & 4 \\
\hline Tinta PVA & $\mathrm{A}_{79}$ & 649,07 & 2 & 1 \\
\hline Verniz Acrílico & $\mathrm{A}_{80}$ & 13965,01 & 15 & 1 \\
\hline $\begin{array}{l}\text { Viga de Peroba de } \\
\text { 6x12cm }\end{array}$ & $\mathrm{A}_{81}$ & 864,00 & 3 & 1 \\
\hline
\end{tabular}




\section{APÊNDICE}

\section{ELECTRE TRI}

O método ELECTRE TRI considera a problemática $\beta($ P. $\beta)$, que classifica as diversas alternativas para a solução de um problema por meio da comparação de cada alternativa potencial com uma referência estável (padrão/alternativa de referência). Para limitar as diversas categorias, são definidas alternativas de referência que são fictícias. Cada categoria tem o limite superior e o inferior restringidos por duas alternativas de referência.

As relações de sobreclassificação S, no ELECTRE TRI, validam ou invalidam a afirmação de que $a \mathrm{~S} b_{\mathrm{h}}$ (e $b_{\mathrm{h}} \mathrm{S} a$ ), o que significa que "a é pelo menos tão boa quanto $b_{\mathrm{h}}$ ", em que $b_{\mathrm{h}}$ são as alternativas de referência. Para que o método possa estabelecer uma relação de sobreclassificação entre uma alternativa a e uma alternativa de referência bh, devem ser calculados os índices de concordância por critério c $\left(a, b_{\mathrm{h}}\right)$ e $\mathrm{c}_{j}\left(b_{\mathrm{h}}, a\right)$; de concordância global $\mathrm{C}\left(a, b_{\mathrm{h}}\right)$ e $\mathrm{C}\left(b_{\mathrm{h}}, a\right)$; de discordância por critério d $\left(a, b_{\mathrm{h}}\right)$ e d $\left(b_{\mathrm{h}}, a\right)$; e de credibilidade $\sigma\left(a, b_{\mathrm{h}}\right)$ (LOURENÇO \& COSTA, 2004). De maneira análoga, o índice de credibilidade $\sigma\left(b_{\mathrm{h}}, a\right)$ permite avaliar como a alternativa $b_{\mathrm{h}}$ supera a alternativa $a$. $\sigma\left(a, b_{\mathrm{h}}\right) . \in[0,1]$ e a afirmação $a \mathrm{Sb}_{\mathrm{h}}$ é considerada válida, se $\sigma\left(a, b_{\mathrm{h}}\right) \geq \lambda\left(\sigma\left(b_{\mathrm{h}}, a\right)\right) \geq \lambda$, em que $\lambda$ é o nível de corte situado no intervalo entre $0,5 \mathrm{e} 1$.

O cálculo de $\mathrm{c}_{j}\left(a, b_{\mathrm{h}}\right)$ é realizado da seguinte forma (MIRANDA \& ALMEIDA, 2003):

$$
c_{j}\left(a, b_{h}\right)= \begin{cases}0 \text { se } g_{j}\left(b_{h}\right)-g_{j}(a) \geq p_{j}\left(b_{h}\right) & \\ 1 \text { se } g_{j}\left(b_{h}\right)-g_{j}(a) \leq q_{j}\left(b_{h}\right) & \\ \frac{p_{j}\left(b_{h}\right)+g_{j}(a)-p_{j}\left(b_{h}\right)}{p_{j}\left(b_{h}\right)-q_{j}\left(b_{h}\right)} & \text { caso contrário }\end{cases}
$$

O mesmo procedimento deve ser usado para calcular $\mathrm{c}_{\mathrm{j}}\left(b_{h}, \mathrm{a}\right)$.

Uma vez calculados os valores dos índices de concordância de cada critério, pode-se realizar o cálculo dos índices globais de concordância, utilizando a fórmula a seguir, em que $\mathrm{k}_{\mathrm{j}}$ é o peso do critério $j$ (MOUSSEAU \& DIAS, 2004; MIRANDA \& ALMEIDA, 2003):

$C\left(a, b_{h}\right)=\frac{\sum_{j=1}^{n} k_{j} c_{j}\left(a, b_{h}\right)}{\sum_{j=1}^{n} k_{j}}$

Para o cálculo do índice de discordância $\mathrm{d}_{\mathrm{j}}\left(\mathrm{a}, b_{\mathrm{h}}\right)$, tem-se que:

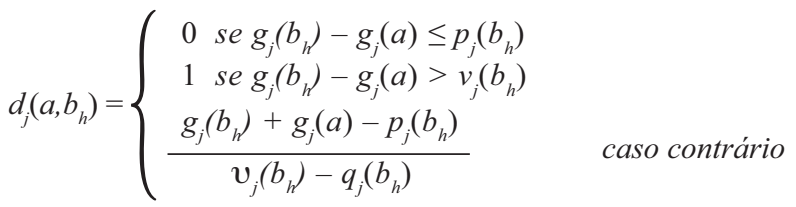

O índice de credibilidade é calculado da seguinte forma:

$\sigma\left(a, b_{h}\right)=C\left(a, b_{h}\right) \prod_{j \in F} \frac{1-d_{j}\left(a, b_{h}\right)}{1-C\left(a, b_{h}\right)}$

onde $\bar{F}=\left\{j \in F: d_{j}\left(a, b_{h}\right)>\mathrm{C}\left(a, b_{h}\right)\right\}$

$\mathrm{O}$ índice de credibilidade de $\sigma\left(\mathrm{b}_{\mathrm{h}}, a\right)$ calcula-se de maneira análoga.

Os valores de $\sigma\left(a, \mathrm{~b}_{\mathrm{h}}\right), \sigma\left(\mathrm{b}_{\mathrm{h}}, a\right)$ e $\lambda$ determinam as situações de preferência entre $a$ e $\mathrm{b}_{\mathrm{h}}$ (MOUSSEAU et al. , 2000):

- $\sigma\left(a, \mathrm{~b}_{\mathrm{h}}\right) \geq \lambda$ e $\sigma\left(\mathrm{b}_{\mathrm{h}}, a\right) \geq \lambda \rightarrow a \mathrm{Sb}_{\mathrm{h}}$ e $\mathrm{b}_{\mathrm{h}} \mathrm{S} a \rightarrow a$ é indiferente $\mathrm{a}_{\mathrm{h}}$;

- $\sigma\left(a, \mathrm{~b}_{\mathrm{h}}\right) \geq \lambda$ e $\sigma\left(\mathrm{b}_{\mathrm{h}}, a\right)<\lambda \rightarrow a \mathrm{Sb}_{\mathrm{h}}$ e não $\mathrm{b}_{\mathrm{h}} \mathrm{S} a \rightarrow a$ é preferível a $\mathrm{b}_{\mathrm{h}}$;

- $\sigma\left(a, \mathrm{~b}_{\mathrm{h}}\right)<\lambda$ e $\sigma\left(\mathrm{b}_{\mathrm{h}}, a\right) \geq \lambda \rightarrow$ não $a \mathrm{Sb}_{\mathrm{h}}$ e $\mathrm{b}_{\mathrm{h}} \mathrm{S} a \rightarrow \mathrm{b}_{\mathrm{h}}$ é preferível a $a$;

- $\sigma\left(a, \mathrm{~b}_{\mathrm{h}}\right)<\lambda$ e $\sigma\left(\mathrm{b}_{\mathrm{h}}, a\right)<\lambda \rightarrow$ não $a \mathrm{Sb}_{\mathrm{h}}$ e não $\mathrm{b}_{\mathrm{h}} \mathrm{S} a \rightarrow a \mathrm{Rb}_{\mathrm{h}}$ e $\mathrm{b}_{\mathrm{h}} \mathrm{R} a \rightarrow a$ é incomparável a $\mathrm{b}_{\mathrm{h}}$.

O procedimento de cálculo credibilidade $\sigma\left(a, \mathrm{~b}_{\mathrm{h}}\right)$ e de $\sigma\left(\mathrm{b}_{\mathrm{h}}, a\right)$ repete-se para cada alternativa de referência $b_{h}$. Deve-se passar, então, ao procedimento de alocação da alternativa a em uma das categorias C predefinidas (GOMES et al., 2004). Tal procedimento divide-se em dois: pessimista e otimista . No pessimista, $a$ é comparado sucessivamente com $\mathrm{b}_{\mathrm{i}}$, para $\mathrm{i}=\mathrm{p}, \mathrm{p}-1, \ldots 0 ; b_{h}$ começa pelo primeiro perfil, tal que $a \mathrm{Sb}_{\mathrm{h}}$, determinando $a$ para a categoria $\mathrm{C}_{\mathrm{h}+1}\left(a \rightarrow \mathrm{C}_{\mathrm{h}+1}\right)$. Já no procedimento otimista, $a$ é comparado sucessivamente $\operatorname{com} b_{i}$, para $\mathrm{i}=1,2, \ldots \mathrm{p} ; b_{h}$ começa pelo primeiro perfil, tal que " $b_{h}$ seja preferível a $a$ ", determinando $a$ para a categoria $\mathrm{Ch}(\mathrm{a} \rightarrow \mathrm{Ch})$. A distinção entre os dois procedimentos reside na seqüência dessa comparação e no critério de identificação da categoria de localização. 